\section{Slow-motion galactic birth}

Joseph Silk

THE timescale over which primordial material collapsed to form our Galaxy is of considerable cosmological significance. The debate is hinged on whether the collapse occurred over a 'dynamical' timescale, which would require all the old stars in the Galaxy to have formed within an initial free-fall time of less than a billion $\left(10^{9}\right)$ years, or whether the early collapse was a more gradual process. Perhaps our Galaxy contracted over severai biiiton years: if this were the case, there are noteworthy, even revolutionary, implications for our picture of galaxy formation. New observations of two globular star clusters by R. J. Dickens et al., reported on page 212 of this issue, strengthen the view that there is a spread of about 3 billion years in the ages of some of the oldest stars in our Galaxy.

The significance of globular clusters, gravitationally bound associations of up to a few million stars, is that they are thought to be the oldest objects in our Galaxy. The two chosen for the new study, NGC288 and NGC362, have long been suspected to differ in age by 3 billion years. This inference is based on the colour-luminosity plot (Hertzsprung-Russell diagram) of the stars in the clusters: specifically, the point at which the stars depart from the main, hydrogen-burning sequence. In older clusters, the more massive, brighter stars will have exhausted their hydrogen cores and only the fainter, less massive stars will remain on the main sequence. The two clusters appear to have the same metallicity, a parameter which affects the turn-off luminosity at a given age and so might thereby introduce further uncertainty into any interpretation of difference in turn-off. This pair of clusters is therefore an ideal candidate for an age difference between two stellar systems that formed when the Galaxy first underwent collanse.

However, previous studies made unwarranted assumptions about the metallicity of these clusters. Astronomers loosely group $\mathrm{C}, \mathrm{N}$ and $\mathrm{O}$, as well as $\mathrm{Fe}$, under the rubric of 'metals', and there is reason to believe that in old stars in the galactic halo, the $\mathrm{O} / \mathrm{Fe}$ abundance may differ from the solar value. Indeed at $[\mathrm{Fe} / \mathrm{H}]<-1$ (a compact notation that means $\mathrm{Fe} / \mathrm{H}$ is less than 10 per cent of the solar value), $[\mathrm{O} / \mathrm{Fe}]$ is about 0.5 (an enhancement of 3 ) relative to the solar value for field halo stars. A factor of 3 increase in $\mathrm{O} / \mathrm{Fe}$ decreases the age by 2 Gyr from that derived for a given turn off assuming the solar $\mathrm{O} / \mathrm{Fe}$ ratio. The previous studies of globular clusters have not directly measured $\mathrm{C}, \mathrm{N}, \mathrm{O}$ and $\mathrm{Fe}$ in the same stars, but assumed a solar ratio of $\mathrm{C}, \mathrm{N}, \mathrm{O}$ to $\mathrm{Fe}$. Moreover, the globular-cluster stars measured are stellar giants whose surface abundances may be contaminated by the nuclear reaction cycle involving $\mathrm{C}, \mathrm{N}$ and $\mathrm{O}$, which occurs in the stellar core. There is therefore serious cause for concern that the ages (and age differences if $\mathrm{O} / \mathrm{Fe}$ varies from cluster to cluster) may be overestimated.

The new study obtains accurate $\mathrm{C}, \mathrm{N}, \mathrm{O}$ and $\mathrm{Fe}$ abundances for samples of stars in the two globular clusters. The $\mathrm{CNO}$ cycle reactions can modify the abundances of $\mathrm{C}, \mathrm{N}$ and $\mathrm{O}$, but the sum $\mathrm{C}+\mathrm{N}+\mathrm{O}$ is conserved. I ne spectroscopic study reveals that although $(\mathrm{C}+\mathrm{N}+\mathrm{O}) / \mathrm{Fe}$ is constant from star to star and from cluster to cluster, $\mathrm{O}$ varies markedly with, for example, C. The variations with $\mathrm{C}$ and with $\mathrm{N}$ in NGC362 confirm the trend expected for material that has undergone $\mathrm{CNO}$ cycling in stellar cores and then been mixed with surface matter. But NGC288 shows no evidence of such surface contamination in its giant stars. The two clusters are inferred to have had very different historics of mixing. The unmixed stars are enhanced in $[\mathrm{O} / \mathrm{Fe}]$ by about 0.2 , a slightly smaller enhancement than seen for halo-field dwarfs at the same metallicity.

The moral of this investigation to observers is: take heed - do not use $\mathrm{O} / \mathrm{Fe}$ in a small sample of stars (an inevitable restriction because of the long integration times needed to acquire high-resolution spectra) to infer global trends of $[(\mathrm{C}+\mathrm{N}+\mathrm{O}) / \mathrm{H}]$, this ratio being the key to age, or relative-age, determinations. Lack of OCEANIC MAGMATISM

\section{Iceland melting dynamics}

\section{Rob Ellam}

RECENT years have seen a transformation in the way petrologists regard melting processes that occur in the Earth's mantle to generate the magmas that crvstallize as basalts at the surface. The breakthrough has come largely from better understanding of the physics of melting, and above all, from the recognition that even rather small melt fractions are likely to form an interconnected network within the partially molten mantle, allowing rapid segregation of melt from the solid matrix ${ }^{1}$. Thus, old ideas of static melt regimes with large melt fractions $(10-20$ per cent by volume) remaining in contact and in chemical equilibrium with the residual solid matrix seem redundant and have been replaced by dynamic melting models in which small melt fractions (less than 2 per cent) are continuously extracted from an upwelling column of mantle ${ }^{2}$. On page 201 of this issue ${ }^{3}$, Elliot et al. present highly coherent major- and trace-element and isotope-ratio variations in Icelandic basalts that appear to indicate the operation of dynamic melting beneath that area. knowledge of $[(\mathrm{C}+\mathrm{N}+\mathrm{O}) / \mathrm{H}]$ made previous attempts at age determinations uncertain by 3 Gyr or more.

Dickens et al. find that the two clusters have almost identical abundances of $[(\mathrm{C}+\mathrm{N}+\mathrm{O}) / \mathrm{H}]$, as well as of $[\mathrm{Fe} / \mathrm{H}]$ and conclude that the clusters therefore do differ

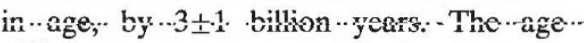
difference comes tantalizingly close to showing that the Galaxy took longer than the free-fall time, about 1 billion years, to collapse. The globular clusters, because of their eccentric orbits, must have formed during this collapse. Gaseous dissipation of the energy released during contraction provides a means of slowing the collapse. Most models of galaxy formation require the round spheroid of the Galaxy to collapse dynamically on a free-fall time. A similar scheme applies to the formation of elliptical galaxies. The longer timescale, if confirmed, should inspire a new approach to galaxy formation theory.

Astronomers are left with another puzzle; the two globular clusters have very different horizontal branch (post-main sequence) morphologies: NGC362 has mostly red giants, whereas NGC288 has predominantly blue giants. A second parameter, other than age, is needed to explain this, and the new study shows that the $[(\mathrm{C}+\mathrm{N}+\mathrm{O}) / \mathrm{Fe}]$ abundance is not the culprit. Theorists and observers alike have much work left to do before the formation of globular clusters is fully understood.

Joseph Silk is in the Departments of Astronomy and Physics, University of California, Berkeley, California 94720, USA.
Two of the most important parameters that control melting processes in the mantle are its temperature and the amount of extension of the lithosphere, the rigid plate above the convecting mantle (asthenosphere). Lithospheric extension allows upwelling of the underlying asthenosphere causing melting through adiabatic decompression. Clearly, for a given extension or stretching factor, the extent of melting will be greatest where the mantle is hottest. Iceland sits astride the North Atlantic ridge where new oceanic lithosphere is created and the stretching factor is therefore effectively infinite. However, beneath Iceland the upwelling mantle is some $200{ }^{\circ} \mathrm{C}$ hotter than that found beneath normal ridge segments. So adiabatic upwelling generates more melt and the most noticeable consequence is the great thickness of the Icelandic crust (not least, the existence of Iceland itself) compared with the average thickness of oceanic crust (typically $7 \mathrm{~km}$; ref. 4).

Melts generated from the hot plume of mantle beneath Iceland are also geochemi- 